\title{
From 2D to 3D: Graphene molding for transparent and flexible probes
}

Cite as: Appl. Phys. Lett. 114, 043301 (2019); https://doi.org/10.1063/1.5075618

Submitted: 23 October 2018 . Accepted: 10 January 2019. Published Online: 28 January 2019

Martin Lee, Thai-An Vuong, Eric Whiteway, (D) Xavier Capaldi, Yuning Zhang, Walter Reisner, Carlos S. RuizVargas, and (D) Michael Hilke

\section{ARTICLES YOU MAY BE INTERESTED IN}

Topographically selective deposition

Applied Physics Letters 114, 043101 (2019); https://doi.org/10.1063/1.5065801

Organic thin film memcapacitors

Applied Physics Letters 114, 043302 (2019); https://doi.org/10.1063/1.5080115

Charge puddles in germanene

Applied Physics Letters 114, 041601 (2019); https://doi.org/10.1063/1.5085304

\section{Hall Effect} Measurement Handbook

A comprehensive resource for researchers Explore theory, methods, sources of errors, and ways to minimize the effects of errors

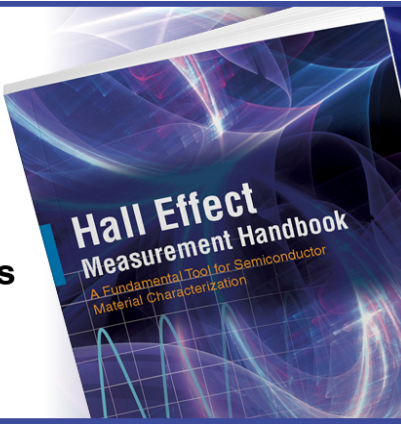

Request it here

Lake Shore
carotronics 


\title{
From 2D to 3D: Graphene molding for transparent and flexible probes
}

\author{
Cite as: Appl. Phys. Lett. 114, 043301 (2019); doi: 10.1063/1.5075618 \\ Submitted: 23 October 2018 - Accepted: 10 January 2019 - Published Online: \\ 28 January 2019 · Corrected: 19 November 2019
}

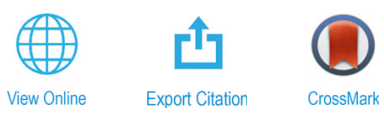

Martin Lee, (D) Thai-An Vuong, Eric Whiteway, Xavier Capaldi, (D) Yuning Zhang, Walter Reisner, Carlos S. Ruiz-Vargas, and Michael Hilke ${ }^{a)}$ (D)

\author{
AFFILIATIONS \\ Center for Physics of Materials, Department of Physics, McGill University, Montreal H3A 2T8, Canada

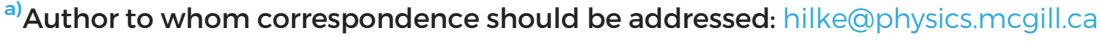

\begin{abstract}
Chemical vapor deposition (CVD) has been widely adopted as the most scalable method to obtain single layer graphene. Incorporating CVD graphene in planar devices can be performed via well-established wet transfer methods or thermal adhesive release. Nevertheless, for applications involving 3D shapes, methods adopted for planar surfaces provide only a crude solution if a continuous, tear-free, wrinkle-free graphene layer is required. In this work, we present the fabrication and characterization of Polydimethylsiloxane-supported 3D graphene probes. To accommodate 3D geometries, we perform CVD on catalysts possessing a non-trivial 3D topology, serving to mold the grown graphene to a final non-trivial 3D shape. This advance overcomes challenges observed in standard transfer processes that can result in uneven coverage, wrinkles, and tears. To demonstrate the potential of our different transfer approach, we apply it to fabricate graphene electrical probes. Graphene, due to its flexibility, transparency, and conductivity, is an ideal material with which conventional metal based probes can be replaced. In particular, with a contact impedance on the order of $10 \mathrm{k} \Omega$, our graphene probes may find applications, such as in electrophysiology studies.
\end{abstract}

Published under license by AIP Publishing. https://doi.org/10.1063/1.5075618

Graphene is a two dimensional carbon allotrope widely known for its remarkable and versatile properties: it is flexible, ${ }^{1}$ stretchable, ${ }^{2}$ conductive ${ }^{3}$ transparent at visible wavelengths, ${ }^{4}$ and yet extremely strong. ${ }^{5}$ Graphene is consequentially an ideal material for flexible electronics. ${ }^{6}$ However, current manufacturing processes are limited to producing flat graphene samples as the catalyst that graphene is grown on is typically obtained in the form of planar substrates. Furthermore, the transfer steps necessary to move graphene from the catalyst surface to the final device complicate the fabrication process and introduce defects from the polymer support layers. ${ }^{8}$ The most widely used graphene transfer process ${ }^{9-11}$ entails coating a layer of poly(methyl-methacrylate) (PMMA) on top of graphene, etching away the catalyst to create a graphene-PMMA stack, transferring the stack to a desired substrate, and dissolving the PMMA with acetone to leave only the graphene. While this process is simple and efficient, it can introduce cracks, wrinkles, contamination, and defects in the transferred graphene ${ }^{12}$ significantly compromising final device quality.

Due to graphene's unique properties, many processes have been developed to incorporate graphene, a two dimensional material, in three dimensional structures. In particular, 3D structures made from graphene foam exhibit higher electrical conductivity than a planar graphene-based composite does and demonstrate great potential as flexible and stretchable conductors. ${ }^{13}$ Composites of 3D graphene foam with inorganic compounds are widely studied, have high reversible capacity and outstanding cycling rate capability in lithium batteries, ${ }^{14}$ and demonstrate remarkable performance as an enzymeless glucose sensor. ${ }^{15}$ However, some fabrication strategies for 3D graphene involve extremely tedious fabrication processes while being exclusive only to a specific purpose. Furthermore, those methods are limited to produce small scale devices and inevitably hinder a wide range of applications. Due to this lack of versatility, these approaches are not suitable for industrial scaling and are consequently limited to research use.

Here, we present an industrially scalable approach for fabricating three dimensional graphene structures. We utilize a catalyst, copper, purchased in the form of a cylindrical tube (so that is already molded into the desired final shape), and perform Chemical vapor deposition (CVD) to grow graphene on the tube surface. We then fill the inside of the tube with a curable 
polymer for structural support and etch away the copper. Using this method, we demonstrate the fabrication of graphene based flexible probes. Furthermore, the geometry facilitates the addition of fibers directly into the probe, providing optical access for combined contact electro-optical applications. Distinct from other approaches where 3D graphene is grown in a foam-like structure, the graphene incorporated in our device is layered graphene, conforming to the device's three dimensional shape. The shape of the copper mold used for the final device is completely flexible. The curable polymer used for structural support can also be chosen freely on an application specific basis. As such, the final device is fully modifiable, providing a wide range of applications.

The fabrication protocol is illustrated in Fig. 1. Copper capillary tubes with an inner diameter of $600 \mu \mathrm{m}$ and an outer diameter of $800 \mu \mathrm{m}$ are cut into $2.5 \mathrm{~cm}$ sections [Fig. 1(a)] using a crimper. The copper tubes are first sonicated in RCA SC-1 solution $\left(1 \mathrm{NH}_{4} \mathrm{OH}: 1 \mathrm{H}_{2} \mathrm{O}_{2}: 5 \mathrm{DI}\right)$ for $30 \mathrm{~min}$. Next, the tubes are rinsed with deionized water (DI), sonicated in $4.2 \% \mathrm{HNO}_{3}$ for $30 \mathrm{~min}$, and rinsed with DI once again. One end of the tubes is then cut at an angle ( $\leq 45^{\circ}$ with respect to the body) which creates a sharp tip [Fig. 1(b)] and rinsed with 2-propanol. The copper tubes are blown dry and placed in our home-made vertically loaded CVD system. The tubes are annealed at $1000^{\circ} \mathrm{C}$ for $\geq 2 \mathrm{~h}$ while flowing $20 \mathrm{sccm}$ of $\mathrm{H}_{2}$ gas. The temperature is then increased to $1050{ }^{\circ} \mathrm{C}$, and $4 \mathrm{sccm}$ of $\mathrm{CH}_{4}$ are introduced. After $1 \mathrm{~h}$ of growth, the tubes are cooled down in $1 \mathrm{~h}$ while maintaining only the $\mathrm{H}_{2}$ gas flow.

Once the graphene coated tubes are removed from the CVD, they are submerged in PMMA A2 in a Nalgene vacuum desiccator to promote a complete coating. We observed that the intermediate PMMA layer between graphene and PDMS
(Polydimethylsiloxane) is necessary because the PMMA A2 is less viscous and therefore conforms better to the surface morphology of the graphene covered copper than PDMS alone. The tubes are then individually spun at $1000 \mathrm{rpm}$ with the open-end pointing out to remove the excess PMMA and ensure a uniform coating $\sim 100 \mathrm{~nm}$. The probes are then baked at $120^{\circ} \mathrm{C}$ for $2 \mathrm{~min}$ and cooled down. The typical soft baking temperature for PMMA is $180^{\circ} \mathrm{C}$ for the purpose of e-beam lithography in order to (1) remove the solvent, (2) to harden the resist, and (3) to enhance the flatness by heating beyond the glass transition temperature. However, for the interest of using PMMA as an intermediate adhesion layer, removing the solvent is the only objective in soft baking. PDMS (Sylgard 184) is mixed (1 curing agent:10 base) and desiccated for $45 \mathrm{~min}$. Once all the bubbles escape, the graphene coated tubes are submerged in the PDMS mix and further desiccated to ensure a void-free structure. After the PDMS settles in the tubes, $100 \mu \mathrm{m}$ optical fibers (Edmund Optics 57-061) are inserted in the copper mold as shown in Fig. 1(c). The tubes are then baked at $80^{\circ} \mathrm{C}$ for $1 \mathrm{~h}$ to cure the PDMS.

Before the copper can be etched, PDMS on the outside of the copper tube is scraped off. Using the optical fiber as support, the tubes are then immersed in a bath of $5 \mathrm{wt}$. \% $\left(\mathrm{NH}_{4}\right)_{2} \mathrm{~S}_{2} \mathrm{O}_{8}$ heated to $80^{\circ} \mathrm{C}$. Once the copper has been fully etched [Fig. $1(\mathrm{~d})]$, the probes are rinsed with DI three times and blown dry. As shown in Fig. 1(e), small amounts of conductive silver epoxy (H2OE EPO-TEK Ted Pella) are used to contact a wire onto the side of the graphene probe body for electrical measurements.

We also provide a possible method for patterning the graphene probe using standard lithography techniques shown in Figs. 1(f)-1(1). Prior to doing so, we characterized the probe using SEM and Raman spectroscopy and measured the probe's electrical impedance.
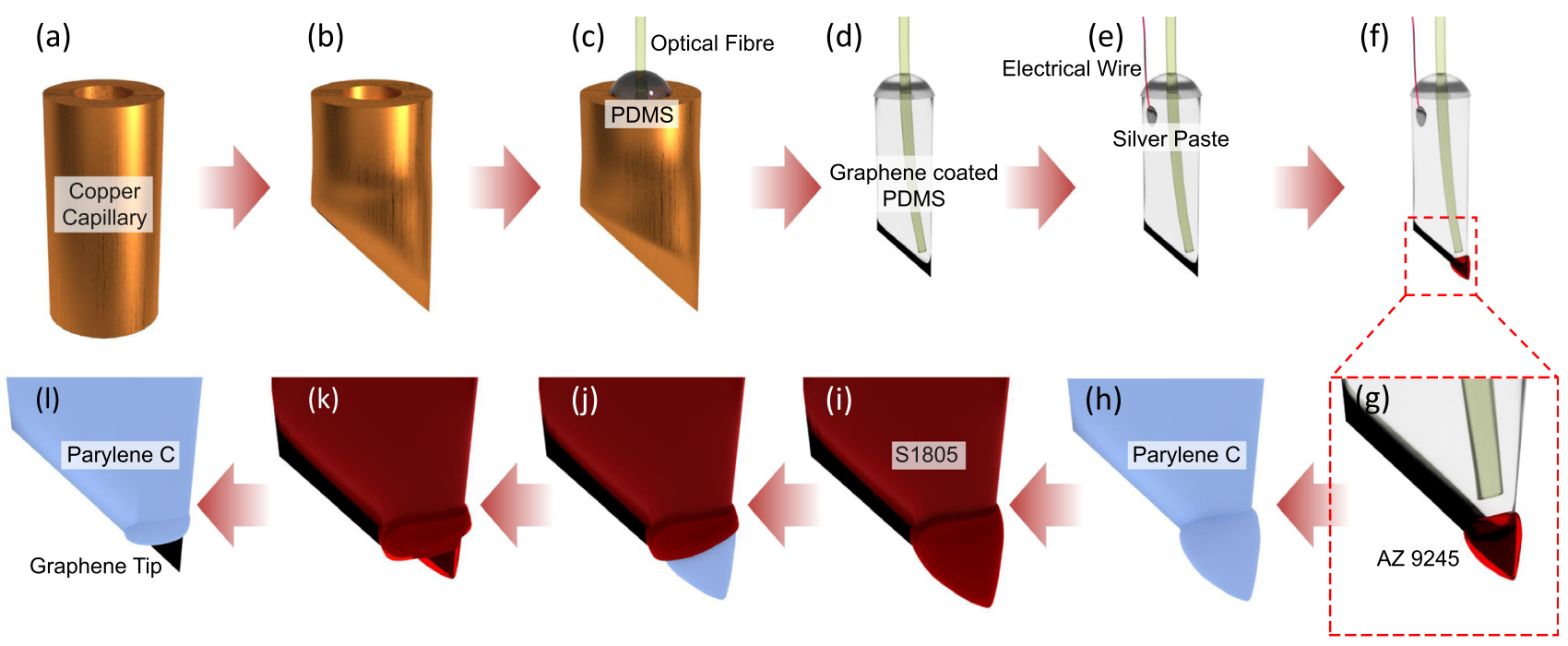

FIG. 1. Illustration of the graphene probe fabrication steps. (a) The starting substrate is a copper capillary tube. (b) One end of the tube is snipped at an angle and graphene is grown on the tube's surface. (c) The tube inside is coated with PMMA and baked and filled with PDMS. After desiccation, an optical fiber is inserted. (d) The copper is etched away in ammonium persulfate. (e) A contacted wire is attached to the graphene via silver epoxy. (f) The tip of the probe is dipped in AZ 9245 using an xyz stage for protection against oxygen plasma. (g) Zoomed in image of (f). (h) Parylene $\mathrm{C}$ is deposited isotropically. (i) The entire probe is coated with S1805 for optical lithography of the tip. (j) The tip is exposed and developed. ( $\mathrm{k}$ ) $\mathrm{O}_{2}$ plasma is used to remove the parylene. (I) The photoresist is removed by acetone. 
The SEM images of the three dimensional structures completely covered with graphene-equivalent to the fabrication step in 1(d)-are displayed in Fig. 2. Figures 2(a) and 2(c) show graphene probes rotated $90^{\circ}$ from each other, (a) showing the graphene probe seen from the cut edge and (c) showing the graphene probe from the side. Respective images in high magnification are shown in (b) and (d). These images show that the graphene probe closely follows the surface contour of the copper mold. The tip of the graphene probe, as seen in Figs. 2(a)-2(c), converges to a point as sharp as a few tens of microns in curvature.

These SEM images show wrinkles and surface roughness of the graphene arising from the copper substrate. This alludes to the retention of the shape of the probe even after the copper is etched away aided by the underlying polymer. Furthermore, a low density of rips and tears is visible under SEM. The lack of a transfer step from the surface of a catalyst to the target substrate helps reduce the formation of defects which can occur during the transfer step.

We further developed a method to insulate the graphene probe while only exposing the tip [Figs. 1(f)-1(1)]. A drop of AZ9245 on a glass slide is placed on a xyz stage, and the probe is hung from above by the optical fiber. The xyz stage is slowly raised until the probe and AZ-9245 are in contact with a meniscus formed at the interface [Figs. 1(f) and 1(g)]. The probe is left to dry for an hour and suspended in the SCS200 parylene coater for a uniform isotropic deposition. One gram of parylene $\mathrm{C}$ dimers is used to deposit $580 \mathrm{~nm}$ parylene on the probe surface [Fig. 1(h)]. After deposition, the probe is dipped in S1805 and suspended to dry for an hour [Fig. 1(i)]. Aluminum foil is used to mask the probe body so that only the $\sim 50 \mu \mathrm{m}$ region around the tip is outside of the $\mathrm{Al}$ foil. An EVG-620 aligner is used to expose
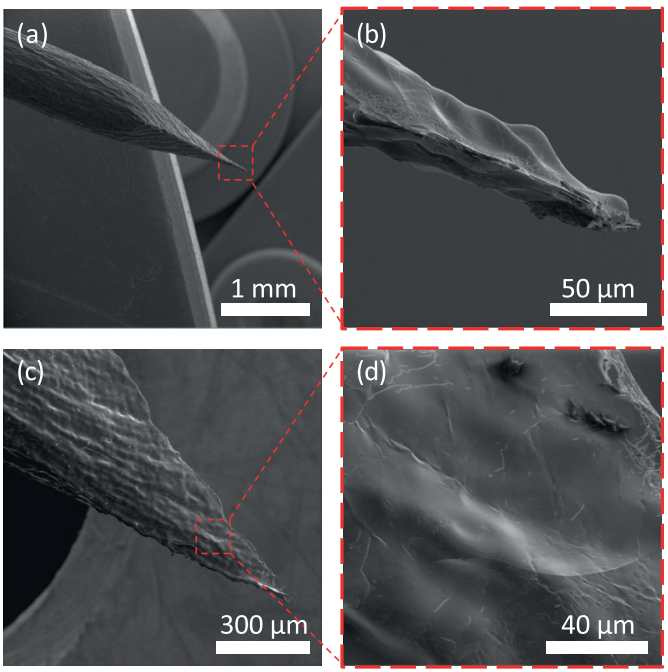

FIG. 2. SEM images of the graphene probe at various magnifications: (a) viewed from the cut edge of the graphene probe and $(b)$ the same at a high magnification and (c) viewed from the side of the graphene probe and (d) the same at a high magnification. the $\mathrm{S} 1805$ coated probe $\left(450 \mathrm{~J} / \mathrm{cm}^{2}\right)$. The probe is then developed in MF-319 for $100 \mathrm{~s}$, revealing the parylene at the tip [Fig. 1(j)]. Using an $\mathrm{O}_{2}$ plasma asher, the parylene at the tip is removed [Fig. 1(k)]. Finally, the probe is submerged in acetone to remove the photoresist masking layer [Fig. 1(1)].

An SEM image of an exposed tip is shown in Fig. 3(b) compared to an illustration in (a). The exposed graphene region is approximately $200 \mu \mathrm{m}$ with no photoresist residue on its surface. We attribute the broadening of the exposed area to the coarse design of our used Al foil photomask. However, a clear separation of boundaries can be observed, which suggests that the patterning method provides sufficient control for the area of the exposed tip.

To reveal the presence and quality of graphene on the tips, we performed Raman spectroscopy. A Renishaw InVia Raman system with a wavelength of $514 \mathrm{~nm}$ was used on the body of the graphene probe. As shown in Fig. 4(a), the Raman spectrum is heavily dominated by the PDMS peaks. However, the PDMS peaks are not in superposition with the $\mathrm{G}$ peak and the $2 \mathrm{D}$ peak of graphene, thus allowing for post-fabrication characterization of graphene using $\mathrm{G}$ and $2 \mathrm{D}$ peaks. The ratio of the $2 \mathrm{D}$ peak and the $\mathrm{G}$ peak is $\sim 0.86$, which suggests that many domains of multilayer graphene are present. ${ }^{16,17}$ This is further supported by the SEM images in Fig. 2. To mask the PDMS contribution, we normalized the total spectrum with the spectrum of an area of the probe not containing graphene. This revealed various peaks of graphene such as the $\mathrm{D}+\mathrm{D}^{\prime \prime}$ peak, $2 \mathrm{D}^{\prime}$ peak, and $2 \mathrm{D}+\mathrm{G}$ peak. ${ }^{18}$ This spectrum was compared with the spectrum from a typical two dimensional graphene on $\mathrm{SiO}_{2} / \mathrm{Si}$ as shown in 4(b). The weakness of the D peak indicates a low density of defects in the graphene. ${ }^{19}$
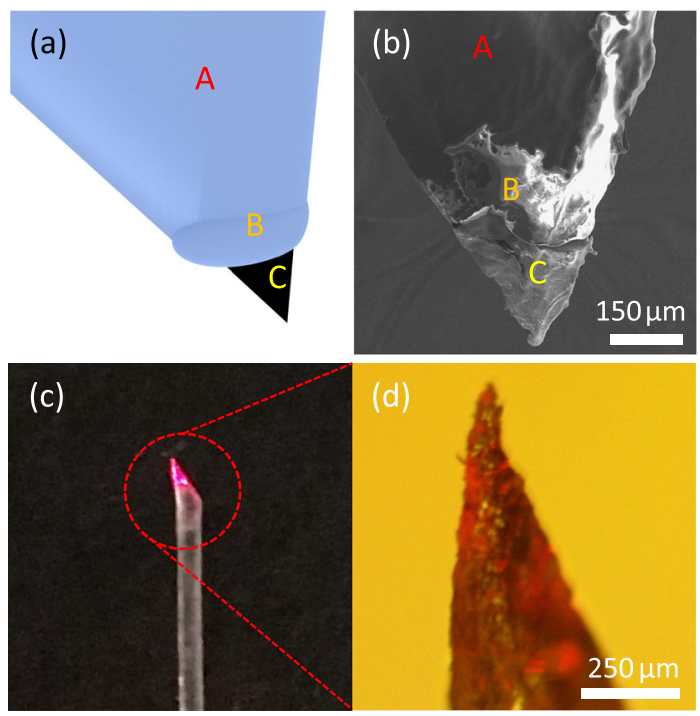

FIG. 3. (a) Illustration of the probe with the exposed tip compared to (b) the actual device. A: Parylene on graphene. B: Parylene on AZ 9245 on graphene. C: Graphene. (c) Optical image of the probe while light with a wavelength of $650 \mathrm{~nm}$ is shone through the optical fiber. A $10 \mathrm{~mW}$ visual fault locator is used as a light source. 


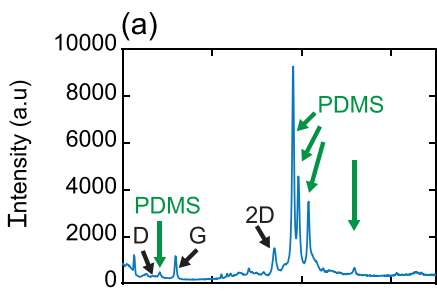

(b)
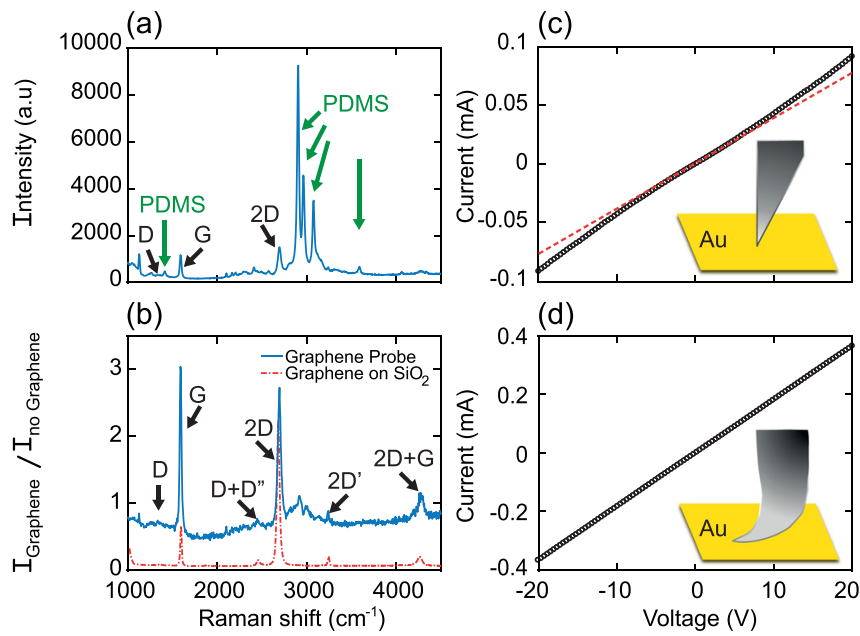

(d)

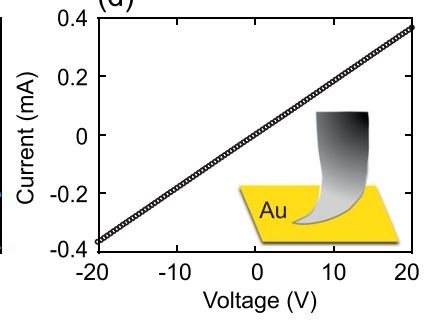

FIG. 4. (a)-(d) Raman spectroscopy performed on the body of the graphene probe and I-V curves. (a) Raman spectrum from the body of the graphene probe before the parylene deposition. (b) A region containing graphene and a region without graphene plotted in a ratio (blue) compared to the averaged Raman spectrum of graphene on $\mathrm{SiO}_{2}$ (red). (c) IV trace of a graphene probe in point contact with a gold pad. A dotted red line is plotted as a visual guide to the linear part. (d) IV trace of a graphene probe in areal contact with a gold pad.

Next, we performed electrical measurements on the graphene probes using a Keithley 2400 sourcemeter. The I-V curves of a graphene probe device are shown in Fig. 4. Probes are brought into contact with a gold pad evaporated on glass, and the voltage is swept while the current is measured. As shown in Fig. 4(c), the probes have a slight non-linear I-V behaviour when only the tip is in contact with the gold pad at low tip-substrate pressure. When greater force is used, the probe tip bends and conforms to the surface of the gold pad, resulting in a larger contact area. The resulting linear I-V trace is shown in Fig. 4(d). These results are based on the fabrication of about 100 tips with a yield close to $100 \%$. The resistance ranges between 15 and $250 \mathrm{k} \Omega$ when the tip barely touches the gold (point contact), while the resistance reaches approximately $10-50 \mathrm{k} \Omega$ when the tip is pushed into the gold surface under pressure (areal contact). After repeated high pressure contacts, the resistance increases and eventually no conductance is measured.

Graphene is grown on the surface of commercially available polycrystalline low purity copper capillary tube. Low purity copper will induce a large number of nucleation sites for the graphene CVD growth. ${ }^{20}$ This leads to faster growth of graphene and also a prevalence of multilayer regions. For most applications, this is not an issue since the conductivity is not strongly affected (doping plays a more dominant role). However, if larger graphene crystals' domain sizes are desired higher purity copper molds can be used. This is relevant for high mobility electronic devices such as Hall sensors, which require high mobilities (the mobility scales close to linearity with the average grain size $\mathrm{e}^{21}$ ). The effect of purity on the mechanical properties is not as well understood. Having multiple multilayer regions can also enhance some mechanical properties. ${ }^{22}$
The mold geometry has the highest impact on our probe quality, as the copper substrate entirely determines the geometry of the resulting device. We formed the sharp probe tip by closing off one side of the capillary copper tube. However, designing a copper substrate with a desired three dimensional shape would provide full control over the probe shape. With the continuously increasing affordability of 3D printers, we believe that graphene molding can easily be implemented in many complex applications, including but not limited to medicine and artificial tissues, ${ }^{23}$ where graphenes' biocompatibility and hemocompatibility properties are needed. ${ }^{24}$ Traditional approaches such as metal injection could also be used to create the copper mold. ${ }^{25}$ The copper quality will determine the graphene growth parameters (such as growth time and graphene characteristics), the copper volume, and the etching time required.

One potential application of our probe is electrophysiology, where rigid metal rods are used to probe the electrical activities of nervous tissues. However, these rigid rods are not able to conform to the tissue of interest, causing swelling of the nearby glial cells or damage to the neurons. ${ }^{26}$ The flexibility of the graphene probe allows for a conformal contact onto the target surface, leading to a lower impedance while reducing the possible damage to nearby tissues. In order to minimize the leakage of current through the ionic solution-which decreases the signal to noise ratio-we provided a possible method of patterning the tip of the graphene probe in Figs. 1(e)-1(1) with the finished product shown in Figs. 3(a) and 3(b).

Furthermore, with the introduction of the optical fiber, our probes can be implemented in optogenetics studies, ${ }^{27}$ delivering optical access simultaneously with electrical access at near single cellular level. Figures 3(c) and 3(d) show optical images of the probe connected to a $650 \mathrm{~nm}$ light source. The optical fiber delivers light to the tip of the probe.

We demonstrated the fabrication and characterization of few layer graphene flexible electro-optical probes using CVD and copper based molding. This process can be generalized to other shapes and for mass production. The graphene layers are largely defect free as evidenced by the weak D peak signal and are composed of a combination of single and a few layers as probed by Raman spectroscopy and scanning electron microscopy. The electrical properties were characterized using I-V measurements, showing close to linear I-Vs. The resistance is stable and varies slightly with contact pressure due to the increased contact area. The resistance is of the order of a few kilo-ohms. Possible applications of these probes include in-vivo electrophysiology, optogenetics, and optical fiber based Raman spectroscopy of nervous tissues using the flexible graphene probe. $^{26}$

We would like to acknowledge the financial support of a joint NSERC, CIHR funded Canadian Health Research Projects grant (CPG-140199).

\section{REFERENCES}

${ }^{1}$ U. Stöberl, U. Wurstbauer, W. Wegscheider, D. Weiss, and J. Eroms, Appl. Phys. Lett. 93, 051906 (2008). 
${ }^{2}$ K. S. Kim, Y. Zhao, H. Jang, S. Y. Lee, J. M. Kim, K. S. Kim, J.-H. Ahn, P. Kim, J.-Y. Choi, and B. H. Hong, Nature 457, 706 (2009).

${ }^{3}$ A. C. Neto, F. Guinea, N. M. Peres, K. S. Novoselov, and A. K. Geim, Rev, Mod. Phys. 81, 109 (2009).

${ }^{4}$ R. R. Nair, P. Blake, A. N. Grigorenko, K. S. Novoselov, T. J. Booth, T. Stauber, N. M. Peres, and A. K. Geim, Science 320, 1308 (2008).

${ }^{5} \mathrm{C}$. Lee, X. Wei, J. W. Kysar, and J. Hone, Science 321, 385 (2008).

${ }^{6}$ D. R. Cooper, B. D'Anjou, N. Ghattamaneni, B. Harack, M. Hilke, A. Horth,

N. Majlis, M. Massicotte, L. Vandsburger, E. Whiteway et al., ISRN Condens. Matter Phys. 2012, 501686.

${ }^{7}$ X. Li, W. Cai, J. An, S. Kim, J. Nah, D. Yang, R. Piner, A. Velamakanni, I. Jung, E. Tutuc et al., Science 324, 1312 (2009).

${ }^{8}$ A. Pirkle, J. Chan, A. Venugopal, D. Hinojos, C. Magnuson, S. McDonnell, L. Colombo, E. Vogel, R. Ruoff, and R. Wallace, Appl. Phys. Lett. 99, 122108 (2011).

${ }^{9}$ X. Li, Y. Zhu, W. Cai, M. Borysiak, B. Han, D. Chen, R. D. Piner, L. Colombo, and R. S. Ruoff, Nano Lett. 9, 4359 (2009).

${ }^{10}$ L. Jiao, B. Fan, X. Xian, Z. Wu, J. Zhang, and Z. Liu, J. Am. Chem. Soc. 130, 12612 (2008).

"A. Reina, H. Son, L. Jiao, B. Fan, M. S. Dresselhaus, Z. Liu, and J. Kong, J. Phys. Chem. C 112, 17741 (2008).

${ }^{12} \mathrm{H}$. Wang and G. Yu, Adv. Mater. 28, 4956 (2016).

${ }^{13}$ Z. Chen, W. Ren, L. Gao, B. Liu, S. Pei, and H.-M. Cheng, Nat. Mater. 10, 424 (2011).
${ }^{14}$ W. Wei, S. Yang, H. Zhou, I. Lieberwirth, X. Feng, and K. Müllen, Adv. Mater. 25, 2909 (2013).

${ }^{15}$ X.-C. Dong, H. Xu, X.-W. Wang, Y.-X. Huang, M. B. Chan-Park, H. Zhang, L.-H. Wang, W. Huang, and P. Chen, ACS Nano 6, 3206 (2012).

${ }^{16}$ D. Graf, F. Molitor, K. Ensslin, C. Stampfer, A. Jungen, C. Hierold, and L. Wirtz, Nano Lett. 7, 238 (2007).

${ }^{17} \mathrm{~V}$. Yu and M. Hilke, Appl. Phys. Lett. 95, 151904 (2009).

${ }^{18}$ S. Bernard, E. Whiteway, V. Yu, D. Austing, and M. Hilke, Phys. Rev. B 86, 085409 (2012).

${ }^{19}$ D. Cai, A. Neyer, R. Kuckuk, and H. M. Heise, J. Mol. Struct. 976, 274 (2010).

${ }^{20} \mathrm{P}$. Braeuninger-Weimer, B. Brennan, A. J. Pollard, and S. Hofmann, Chem. Mater. 28, 8905 (2016).

${ }^{21}$ D. Van Tuan, J. Kotakoski, T. Louvet, F. Ortmann, J. C. Meyer, and S. Roche, Nano Lett. 13, 1730 (2013).

${ }^{22}$ K. S. Choi, F. Liu, J. S. Choi, and T. S. Seo, Langmuir 26, 12902 (2010).

${ }^{23}$ R. Podila, T. Moore, F. Alexis, and A. M. Rao, RSC Adv. 3, 1660 (2013).

${ }^{24}$ A. M. Pinto, I. C. Goncalves, and F. D. Magalhães, Colloids Surf., B 111, 188 (2013).

${ }^{25}$ L. Moballegh, J. Morshedian, and M. Esfandeh, Mater. Lett. 59, 2832 (2005).

${ }^{26}$ W. M. Grill, Expert Rev. Med. Devices 2, 409 (2005).

${ }^{27}$ A. M. Aravanis, L.-P. Wang, F. Zhang, L. A. Meltzer, M. Z. Mogri, M. B. Schneider, and K. Deisseroth, J. Neural Eng. 4, S143 (2007). 